\title{
Surface Morphology of High-speed Spun Poly (ethylene terephthalate) Fibers
}

\author{
Yutaka Kawahara $^{* \dagger}$, Masayoshi Ohara ${ }^{* \star}$, Masaki Tsuji ${ }^{* \star}$ and Takeshi Kikutani ${ }^{* \star \star}$ \\ * Division of Advanced Fibro-Science, Graduate School, Kyoto Institute of Technology, Matsugasaki, Sakyo-ku, Kyoto 606- \\ 8585 Japan \\ ** Laboratory of Polymer Condensed States, Division of States and Structures III, Institute for Chemical Research, Kyoto \\ University, Uji, Kyoto-Fu 611-0011 Japan \\ *** Department of Organic and Polymeric Materials, Tokyo Institute of Technology, O-okayama, Meguro-ku, Tokyo 152-8552 \\ Japan
}

Received 24 August 1999; received 26 January 2000

\begin{abstract}
Permanganic etching was performed on the high-speed spun and regular fibers of poly (ethylene terephthalate)(PET), and their surface morphologies were investigated using a transmission electron microscope. The high-speed spun PET fibers which have low molecular orientation in the amorphous regions showed peculiar surface morphology. That is, lots of small voids reflecting the disordered amorphous regions were observed. However, the number of such voids was extremely small for the regular PET fibers which have well oriented amorphous regions.
\end{abstract}

Keywords: Poly (ethylene terephthalate); Permanganic etching; Morphology

\section{Introduction}

It is well known that some kinds of poly (ethylene terephthalate)(PET) fibers spun by high-speed melt spinning show the higher dyeability in the conventional dyeing process using water at a temperature of lower than $100^{\circ} \mathrm{C}$ $[1],[2]$. The lower molecular orientation in the amorphous regions of the high-speed spun PET fibers promotes the diffusion of dye molecules into the fibers, because the free volume mechanism is dominant in polyester dyeing ${ }^{[3]}$.

Therefore, it is interesting to clear the difference in the surface morphology as well as fiber structure between the high-speed spun and so-called regular PET fibers.

\section{Experimental}

The high-speed spun PET fibers were made as follows. Molten polymer was extruded through a spinneret of $1 \mathrm{~mm}$ diameter at a mass out flow rate of $1.5 \mathrm{~g} / \mathrm{min}$ and was spun at a speed of $6 \mathrm{~km} / \mathrm{min}$ by the take-up device located at 330 $\mathrm{cm}$ below the spinneret. The spin line cooling device was settled below the spinneret, and the ambient temperature there was controlled to be $-50{ }^{\circ} \mathrm{C}$. The regular PET fiber ( $75 \mathrm{~d}, 24$ filaments, Toyobo) was purchased for comparison.

Refractive indices parallel $\left(\mathrm{n}_{\|}\right)$and perpendicular $\left(\mathrm{n}_{\perp}\right)$ to the fiber axis were measured using an interference microscope (Carl Zeiss, Jena) equipped with a polarizing filter. The birefringence was calculated as the difference between these two refractive indices. The Lorentz density (LD) which has a linear relation with the density was calculated by the equation,

$L D=\left(n^{2}-1\right) /\left(n^{2}+2\right)$, where $n^{2}=\left(n_{\|}^{2}+2 n_{\perp}^{2}\right) / 3$.

The differential scanning calorimetry (DSC) measurement was performed using DSC8230 (Rigakudenki) at a heating rate of $10 \mathrm{~K} / \mathrm{min}$ in a nitrogen gas atmosphere. Prior to the measurement, the fibers were cut to powder-like short fiber. The weight of the sample was about $3 \mathrm{mg}$.

The samples used for X-ray diffraction were prepared by forming the fibers into a bundle. By adjusting the mass of the sample, the volume of the sample irradiated by the X-ray was kept approximately constant. Wide-angle X-ray diffraction (WAXD) was measured with an imaging plate RAXIS DS3 (Rigakudenki) using CuK ${ }_{\alpha}$ X-rays monochromatized by graphite and collimated by two pinholes. The crystallite sizes were estimated using Scherrer's equation.

A permanganic etchant ${ }^{[4]}$ was made at $30^{\circ} \mathrm{C}$ for $30 \mathrm{~min}$ with a solution of $1 \mathrm{wt} \%$ potassium permanganate dissolved in a mixture of $2: 1 \mathrm{H}_{3} \mathrm{PO}_{4}: \mathrm{H}_{2} \mathrm{SO}_{4}$. The two-stage carbon replica $^{[5]}$ of the surface of the PET fibers treated by permanganic etching was prepared using collodion and by Pt-Pd shadowing. The replica thus prepared was examined with a transmission electron microscope (TEM; JEOL, JEM$100 \mathrm{U})$, which was operated at $80 \mathrm{kV}$.

\section{Results and Discussion}

\footnotetext{
${ }^{\dagger}$ Corresponding author Fax:+81-75-724-7337, E-mail: kawahara@ipc.kit.ac.jp
} 


\subsection{Physical Properties of PET Fiber}

The WAXD patterns suggested that both PET fibers have well-developed crystalline structure, that is, high crystallinity and high molecular orientation of crystalline regions. In Table 1 , the crystallite size, birefringence, Lorentz density, crystallinity, and melting point temperature for these PET fibers are listed. It is well known that the crystallite sizes of PET fibers increase with increasing the spinning velocity since the crystallization temperature on the high-speed spinning line becomes higher with increasing the spinning velocity ${ }^{[1],[6]}$. Thus, the melting point temperature of high-speed spun PET fibers became higher. The DSC thermogram for these PET fibers only showed an endothermic peak. From the area of endothermic peak, the crystallinities for these fibers were estimated. The value of $121 \mathrm{~J} / \mathrm{g}$ was used for the heat of fusion of perfect crystalline $\mathrm{PET}^{[7]}$. The most remarkable difference between these PET fibers from the structural point of view is that the high-speed spun PET fibers have a lower birefringence value but have larger crystalline regions as compared with the regular PET fibers. This suggests that the molecular orientation in the amorphous regions of high-speed spun PET fibers is extremely lower than that in the regular PET fibers.

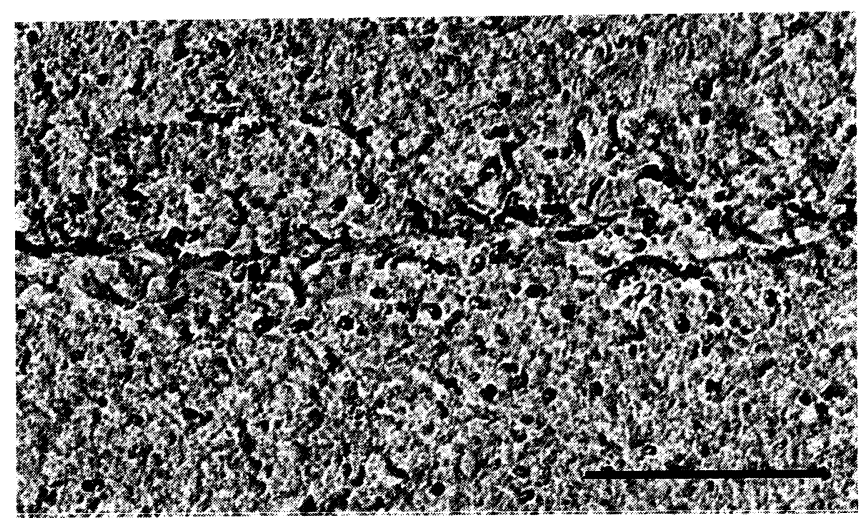

Fig. 1 TEM image of the two-stage surface replica for 6-1.5$\mathrm{C}$ fibers. Fiber axis is horizontal. Scale bar $=0.5 \mu \mathrm{m}$.

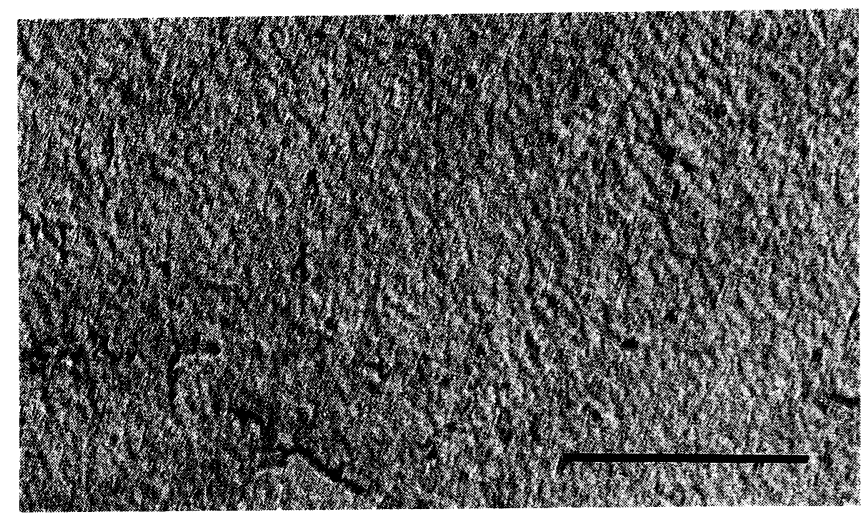

Fig. 2 TEM image of the two-stage surface replica for regular fibers. Fiber axis is horizontal. Scale bar $=0.5 \mu \mathrm{m}$.
Table 1. Physical Properties of PET Fiber

\begin{tabular}{ccc}
\hline \hline & High-speed spun & Regular \\
\hline Diameter $(\mu \mathrm{m})$ & 15.2 & 18.0 \\
Crystallite size $(\mathrm{nm})$ & & \\
L010 & 5.93 & 4.80 \\
L110 & 4.78 & 4.19 \\
L100 & 4.68 & 3.08 \\
L105 & 6.12 & 4.94 \\
Birefringence & 0.1024 & 0.1361 \\
Lorentz density & 0.3427 & 0.3413 \\
Crystallinity (\%) & 49.8 & 36.1 \\
Melting point ( $\left.{ }^{\circ} \mathrm{C}\right)$ & 266.1 & 245.1 \\
\hline
\end{tabular}

\subsection{Surface Morphology}

Permanganic etching was performed on the PET fibers, and then the surface morphologies were investigated using a TEM. It is known that the permanganic etchant preferentially removes more disordered material, revealing the semicrystalline morphology ${ }^{[4]}$. In Fig. 1, a TEM image of the two-stage surface replica for the high-speed spun PET fibers is shown. Lots of small voids are observed. These voids reflect the disordered amorphous regions which will influence the dyeing behavior. On the contrary, for a TEM image of regular PET fibers in Fig. 2, the number of such voids was extremely small. This is closely related to the fact that the regular PET fiber have well-oriented amorphous regions as compared with the high-speed spun PET fibers(see Table 1). Therefore, in order to increase the dye exhaustion for regular PET fibers, it seems necessary to disorder the amorphous regions by elevating the dyeing temperature.

\section{References}

[1] Y. Murase and A. Nagai, "Advanced fiber spinning technology", Ed. by T. Nakajima, Woodhead Publishing, Cambridge, England, pp.25-63, 1994.

[2] Y.Kawahara, H J Jeon, and T Kikutani, J.S.D.C., 115, 355(1999).

[3] T. Hori and H. Zollinger, Text.Chem.Color., 18(10), 19(1986).

[4] S. D. Hudson and A. J. Lovinger, Polymer, 34, 1123(1993).

[5] M.Tsuji, "Comprehensive Polymer Science", Ed. by Sir G.Allen and J.C.Bevington, Vol.1, Pergamon, Oxford, Chapt.34, pp.785-840(1989).

[6] T.Kikutani, Y.Kawahara, T.Matsui, A.Takaku, and J.Shimizu, Seikei-Kakou, 1, 333(1989).

[7] R.C.Roberts, Polymer, 10, 113(1969). 